\title{
Single isotropic 3D fast spin echo sequence compared with conventional 2D sequences for detecting meniscal and cruciate ligament tears in the knee
}

\author{
Nevien Ezzat El-Liethy ${ }^{*^{*}}$ (D, Amr Samir Rashwan² and Heba Kamal ${ }^{1}$ (B)
}

\begin{abstract}
Background: This work was conducted to assess the diagnostic efficiency of isotropic three-dimensional VISTA-fast spin echo versus standard two-dimensional fast spin echo at $1.5 \mathrm{~T} \mathrm{MRI}$, in the assessment of internal knee derangement in symptomatic patients, aiming to obtain similar diagnostic accuracy in a shorter time span, with reduction of partial volume artifacts by thin continuous sections.

Results: This was a non-randomized control study including 39 patients (32 male and 7 females, mean age 37 years old). A correlative study was done utilizing MRI standard 2D FSE (protocol A) versus 3D-VISTA-FSE (protocol B) for medial meniscus (MM) and lateral meniscus $(L M)$, as well as anterior cruciate ligament $A C L$ lesions, comparing the MRI results with the findings of arthroscopy as the gold standard. Both protocols depicted medial meniscus lesions with accuracy, specificity, and sensitivity $(97.44 \%, 96.30 \%$, and 100\% respectively), lateral meniscus lesions with accuracy, specificity, and sensitivity $(97.44 \%, 100 \%$, and 50\% respectively), and ACL lesions with accuracy, specificity, and sensitivity $(100 \%, 100 \%$, and $100 \%$ respectively), while there were no PCL lesions depicted through the study population. Comparing the time factor between both protocols revealed protocol A to consume 13.7 min, while protocol B consumed 6.6 min.

Conclusion: Three-dimensional isotropic VISTA-FSE sequence, although having similar accuracy in diagnosing cruciate and meniscal lesions as the standard sequences, facilitates thin-section data acquisition and multi-planar image reformation in standard and non-standard planes, without intersection gaps that are crucial for the detection and dissection of compound structures; also, it allows a shorter time span, which is more advantageous for patients, particularly the traumatized and emergency patients.
\end{abstract}

Keywords: Knee MRI, 3D versus 2D FSE, Meniscal and cruciate lesions

PACS Picture archiving and communication system

SD Standard deviation

MPR Multi-planar reformation

IW Intermediate weighted

SPSS Statistical Package for the Social Science
Lt Left

Rt Right

PHMM Posterior horn medial meniscus

PHLM Posterior horn lateral meniscus

* Correspondence: nevienelliethy@yahoo.com

${ }^{1}$ Department of Diagnostic and Interventional Radiology, Kasr Al-Aini, Faculty of Medicine, Cairo, Egypt

Full list of author information is available at the end of the article

\section{Background}

Magnetic resonance imaging (MRI) is a specific, detailed, and noninvasive method that is used to assess internal derangements of the knee [1].

\section{Springer Open}

(c) The Author(s). 2020 Open Access This article is licensed under a Creative Commons Attribution 4.0 International License, which permits use, sharing, adaptation, distribution and reproduction in any medium or format, as long as you give appropriate credit to the original author(s) and the source, provide a link to the Creative Commons licence, and indicate if changes were made. The images or other third party material in this article are included in the article's Creative Commons licence, unless indicated otherwise in a credit line to the material. If material is not included in the article's Creative Commons licence and your intended use is not permitted by statutory regulation or exceeds the permitted use, you will need to obtain permission directly from the copyright holder. To view a copy of this licence, visit http://creativecommons.org/licenses/by/4.0/. 
The regular MRI technique for evaluation of internal derangements of the knee utilizes two-dimensional (2D) fast spin echo (FSE) sequence, because of its higher contrast and signal-to-noise ratio. This sequence is satisfactory in evaluating joint abnormalities such as ligamentous injuries, fibro-cartilage damage, and meniscal tears [2].

Current standard 2D MR imaging has several limitations, such as (a) rather thick image sections, associated with gaps in-between, which can obscure fine anatomical details due to partial volume artifacts; (b) non-isotropic voxels; and (c) they are almost repeated in multiple planes when used to evaluate the knee joint, as reconstructions are not possible, thus extending examination time [3].

Isotropic 3D MR images can be performed with a size of $0.5 \times 0.5 \times 0.5 \mathrm{~mm}$ voxels. This can minimize the partial volume artifacts seen with conventional 2D MR images [4]. Multi-planar reformatting can be applied in all necessary planes, including the standard orthogonal planes, within only a few seconds. Hence, it can be helpful for the detection and diagnosis of complex or abnormally positioned internal structures of the knee. This imaging sequence have better diagnostic performance in detecting small radial and root tears of the menisci than conventional 2D images [5]. This was presumably due to a smaller interslice gap and the ability to reconstruct images along the structures of concern [2].

MRI of the knee is known to have high sensitivity and specificity for the detection of ACL ligament and meniscal tears. The 3D VISTA sequence can afford highresolution volumetric images. Acquisition time and inter-echo spacing are optimized by applying reduced flip angles in conjunction with non-selective refocusing pulses. Combining parallel acquisitions utilizing robust factors in both directions of the encoding phase establishes faster acquisition times [2].

The purpose of this study was to demonstrate the added value, diagnostic accuracy, and superiority of isotropic three-dimensional (3D) fast spin echo over standard two-dimensional (2D) fast spin echo at $1.5 \mathrm{~T}$ MR sequences in the interpretation of meniscal and cruciate ligament lesions in symptomatic patients.

\section{Methods}

\section{Patients}

We designed a non-randomized control study for patients clinically presenting with persistent chronic knee pain or post traumatic status, who were referred from the orthopedic outpatient clinic to the Radiology Department, during the time period February 2017 to February 2018. The study included 39 patients ( 32 male and $7 \mathrm{fe}-$ males), which age ranged from 15-60 years (mean age 37 years). All patients included in the study had MRI examination, including both protocols (A and B), followed by arthroscopy as the gold standard reference. A correlative study was conducted between the MRI results and arthroscopic findings. Patients who were excluded from the study include those who had previous surgery on the same knee, when the interval between MRI study and arthroscopy exceeded 21 days, patients with poor image quality due to any type of artifact, those with acute knee fracture, and patients with isolated degenerative meniscal or cruciate ligament lesions, as they were not candidate for arthroscopy (gold standard). Written informed consents were collected from all patients.

\section{MR protocol}

MRI examinations were done on "ACHIEVA $1.5 \mathrm{~T}$ equipment (from PHILIPS Medical Systems, Best, the Netherlands)" utilizing a phased array knee coil. The candidate lied supine with joint space in the middle of the coil, while the knee joint was maintained in extension with slight flexion.

The standard knee protocol (coronal T1, axial T2, and sagittal proton density) was designated protocol $\mathrm{A}$, while the 3D PD-FS (FSE) imaging was designated protocol $\mathrm{B}$ (Table 1).

\section{Imaging analysis and interpretation}

The produced MR images were transferred to a workstation using the Digital Imaging and Communications in Medicine (DICOM) format. Two musculoskeletal radiologists, each with an experience in interpretation of knee MR images more than 10 years, individually studied the

Table 1 Protocol of MR imaging

\begin{tabular}{|c|c|c|c|c|c|c|c|c|c|}
\hline & TR & TE & FOV & SEC, THICK & GAP & Matrix & No of se. & Rcoms. voxel size (mm) & Image time (min) \\
\hline \multicolumn{10}{|l|}{ Standard protocol (A) } \\
\hline Coronal T1 (TSE) & 403 & 7.6 & 160 & 4 & 1 & $516 \times 320$ & 22 & NA & 4.43 \\
\hline Axial T2 (TSE) & 2904.9 & 111.6 & 160 & 4 & 1 & $516 \times 320$ & 22 & NA & 3.18 \\
\hline Sagittal PD (TSE) & 5000 & 30 & 180 & 4.5 & 1 & $512 \times 256$ & 75 & NA & 6.15 \\
\hline \multicolumn{10}{|l|}{ Protocol (B) } \\
\hline Sagittal PD-VISTA FS & 1600 & 33 & 160 & 0.5 & 0 & $320 \times 320$ & 300 & $0.5 \times 0.5 \times 0.5$ & 6.62 \\
\hline
\end{tabular}

NA not applicable, VISTA volume isotropic turbo spin echo acquisition, FS fat-suppressed 
Table 2 Comparative study between protocol A and protocol B

\begin{tabular}{|c|c|c|c|c|c|c|c|c|c|c|c|c|c|}
\hline Item & TP & FN & TN & FP & SEN. & $95 \% \mathrm{Cl}$ & SPEC. & $95 \% \mathrm{Cl}$ & (+) PV & $95 \% \mathrm{Cl}$ & (-) PV & $95 \% \mathrm{Cl}$ & ACC. \\
\hline MRI 2DMM & 12 & 0 & 26 & 1 & 100.00 & 73.535 to 100.000 & 96.30 & 81.029 to 99.906 & 92.31 & 34.979 to 96.183 & 100.00 & & 97.44 \\
\hline MRI 2D LM & 1 & 1 & 37 & 0 & 50.00 & 1.258 to 98.742 & 100 & 90.511 to 100 & 100.00 & 8.476 to 91.524 & 97.37 & 90.238 to 99.329 & 97.44 \\
\hline $\mathrm{MRI} 2 \mathrm{D} \mathrm{ACL}$ & 21 & 0 & 18 & 0 & 100.00 & 83.890 to 100.000 & 100.00 & 81.470 to 100.000 & 100.00 & & 100.00 & & 100.00 \\
\hline MRI 2D PCL & 0 & 0 & 39 & 0 & & & 100.00 & 90.975 to 100.000 & 100.00 & & 100.00 & & 100.00 \\
\hline MRI 3D MM & 12 & 0 & 26 & 1 & 100.00 & 73.535 to 100.000 & 96.30 & 81.029 to 99.906 & 92.31 & 63.681 to 98.797 & 100.00 & & 97.44 \\
\hline MRI 3D LM & 1 & 1 & 37 & 0 & 50.00 & 1.258 to 98.742 & 100.00 & 90.511 to 100.000 & 100.00 & & 97.37 & 90.247 to 99.329 & 97.44 \\
\hline MRI 3D ACL & 21 & 0 & 18 & 0 & 100.00 & 83.890 to 100.000 & 100.00 & 81.470 to 100.000 & 100.00 & & 100.00 & & 100.00 \\
\hline MRI 3D PCL & 0 & 0 & 39 & 0 & & & 100.00 & 90.975 to 100.000 & 100.00 & & 100.00 & & \\
\hline
\end{tabular}

two sets of MR protocols, each one blinded from the other, and from the arthroscopic findings, the 3D images were generated concurrently during image revision by the use of PACS software, and then they correlated their results with each other and with the results of arthroscopy. For each examined joint, the following were assessed and reported in both protocols (A and B).

The anterior ligament ACL was assessed either by the presence or absence of tears (partial or complete). The diagnostic findings of cruciate ligament tear were an abnormal course, increased signal intensity or discontinuity, and indistinct margins with or without abnormal signal [6].

The menisci The medial and lateral menisci were assessed (body, anterior and posterior horns). The criteria for meniscal tear were increased signal intensity within the meniscus reaching the meniscal articular surfaces or abnormal meniscus morphology [6].

Reformation of the isotropic 3D MR images and image analysis were performed simultaneously with picture archiving and communication system software. Reformatted images with $0.05 \mathrm{~cm}$ section thickness in axial and coronal planes were formed semi-automatically.

\section{Arthroscopic interpretation}

Arthroscopies were done by specialized orthopedic surgeons, with experience in knee joint intervention more than 10 years. Arthroscopies were done within average time interval of $14 \pm 7$ days to the MR studies. At arthroscopy, each cruciate ligament was classified as being normal or having partial or full thickness tear. Signs of
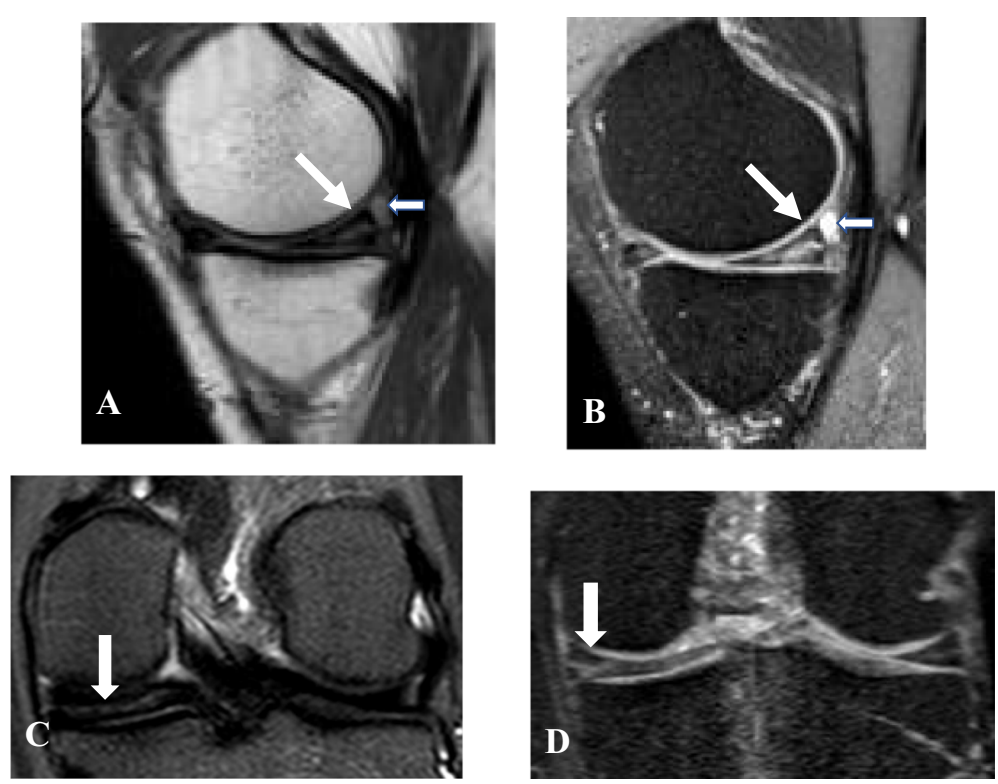

Fig. 1 A 32-year-old male patient presented with acute LT knee joint pain after trauma. a, b Sagittal standard 2D PDWI and 3D MPR VISTA images show horizontally oriented tear of the medial meniscus posterior horn (PHMM) (white arrows) with small related parameniscal cyst, with the tear clearly outlined in the VISTA images. $\mathbf{c}$, $\mathbf{d}$ Coronal standard 2D PD and 3D MPR VISTA images again show the horizontal tear of the PHMM (white arrows) 

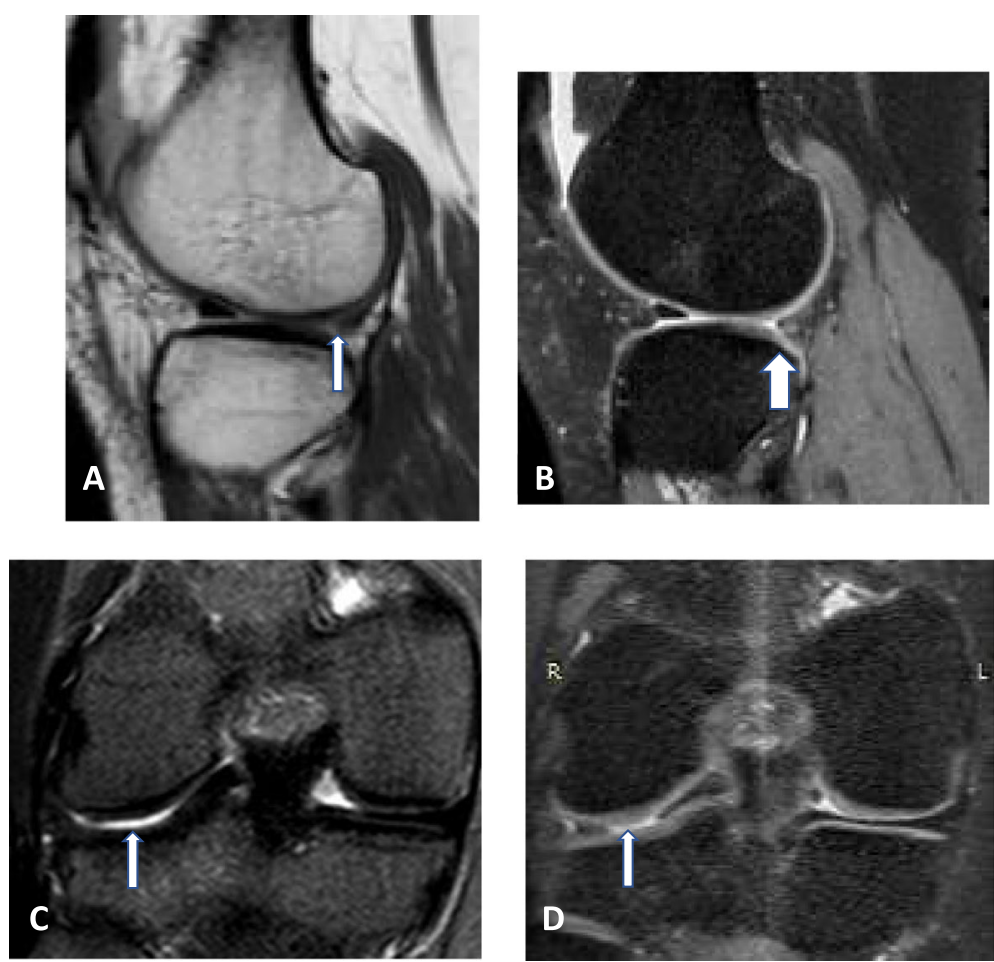

Fig. 2 A 27-year-old male patient presented by acute RT knee joint pain after trauma. a, b Sagittal standard 2D PDWI and 3D MPR VISTA. c, d Coronal standard 2D T2 and 3D MPR VISTA images show lateral meniscus bucket handle tear (white arrows), which is more clear at the 3D MPR VISTA images
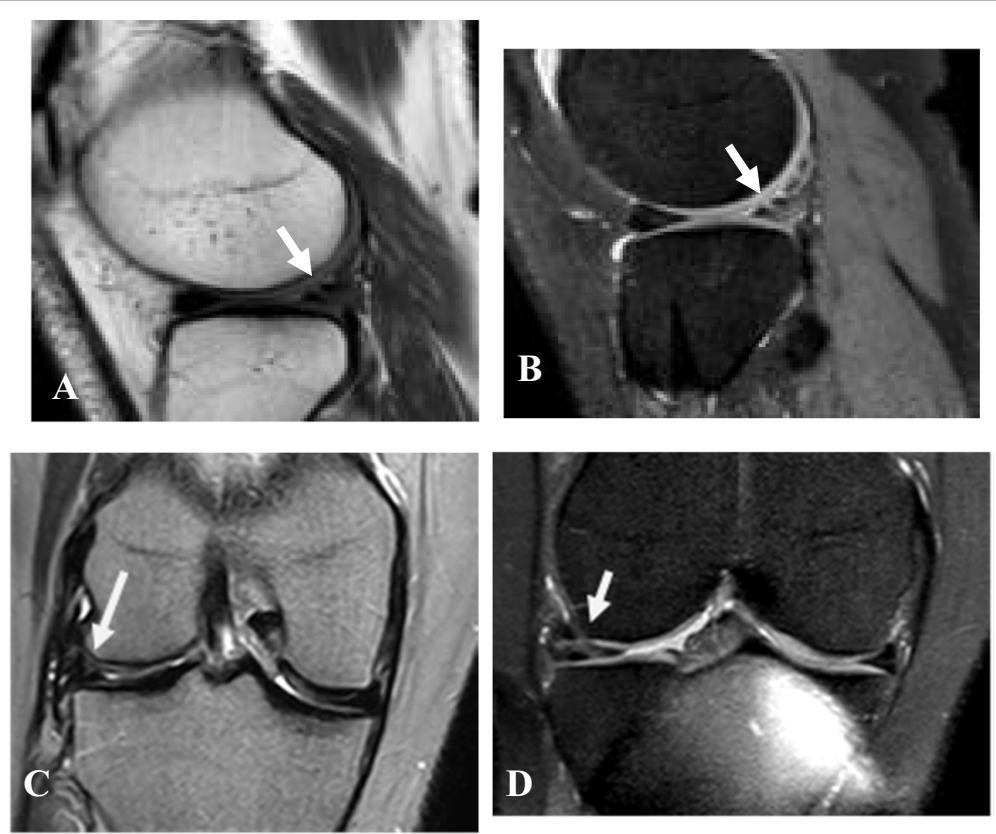

Fig. 3 A 29-year-old male patient presented by acute RT knee joint pain after trauma. a, b Sagittal Standard 2D PDWI and 3D MPR VISTA images show complex branching tear in PHLM (white arrows). c, d Coronal standard 2D PD and 3D MPR VISTA images again show complex branching tear in PHLM (long white arrows), clearly outlined in the VISTA images 

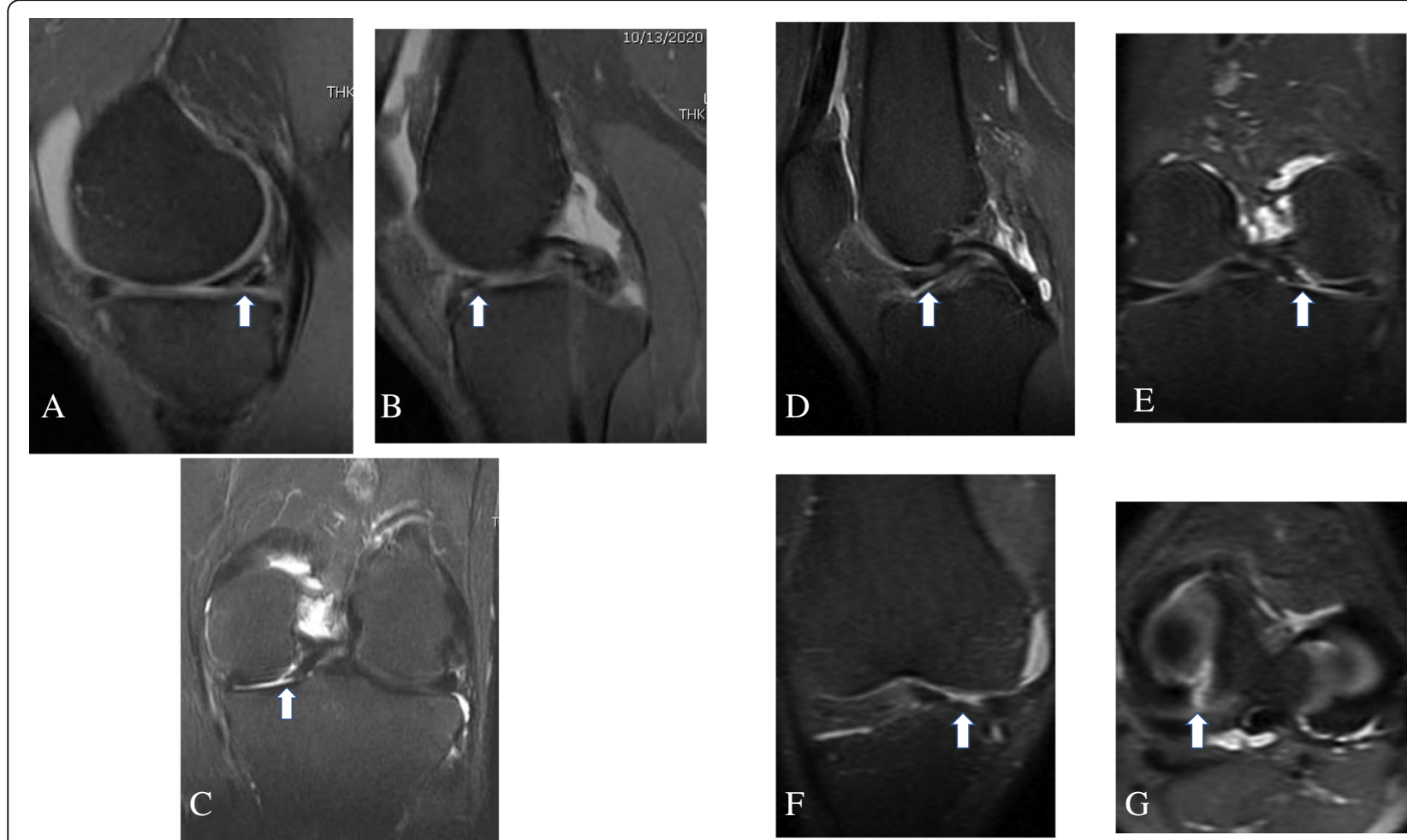

Fig. 4 A 35-year-old male patient presented by acute left knee pain after trauma. a, b Sagittal standard 2D PD WI and c coronal standard 2D PD WI, showing bucket handle tear at the PHMM, arrowed at $\mathbf{a}$ and $\mathbf{c}$, with flipped meniscal fragment anteriorly posterior to the anterior horn, arrowed at $\mathbf{b}$. $\mathbf{d}-\mathbf{g}$ 3D MPR VISTA images (sagittal, coronal, and axial), showing the flipped triangular meniscal fragment, arrowed at $\mathbf{d}$ and $\mathbf{f}$ and the bucket handle tear arrowed at $\mathbf{e}$ and $\mathbf{g}$

existing tear include decreased tension on probing, grossly or focally disrupted bundle. Each meniscus was classified as either intact or torn, a visible fissure extending through the meniscal substance, or just surface fraying, which were all signs of meniscal pathology.

\section{Statistical studies}

Obtained data were statistically analyzed in the form of range, mean, standard deviation $( \pm \mathrm{SD})$, frequencies (number of cases), and percentages.

Categorical data comparison was done by using chisquare test. Exact test was used instead when the expected frequency is less than 5. Agreement between 2D and $3 \mathrm{D}$ findings was obtained using kappa statistic.

Accuracy was represented by the terms of sensitivity, specificity, positive $(+\mathrm{ve})$ predictive value, negative (-ve) predictive value, and overall accuracy. All calculations were performed by computer programs Microsoft Excel 2003 (Microsoft Corporation, USA) and SPSS (Statistical Package for the Social Science; SPSS Inc., Chicago, USA) version 15 for Microsoft Windows.

Screening test done using MedCalc version 18.9. Fisher exact correction was done for comparison of qualitative variables.

\section{The standard of reference}

Knee arthroscopy was considered the gold standard technique.

\section{Results}

Diagnostic performance of both protocols

Comparative study was performed between MRI protocol A versus protocol B for different meniscal and cruciate ligament lesions, and then compared with arthroscopic findings (Table 2).

\section{Meniscal lesions (Figs. 1, 2, 3, 4, and 5) (Tables 3, 4, 5, and 6)}

Both MRI protocols detected medial meniscus injury in 13 knee joints while arthroscopy detected it in 12 knee joints; both modalities agreed in 12 patients, establishing a sensitivity $=100 \%$, specificity $=96.30 \%$, positive predictive value $=92.31 \%$, negative predictive value $=100 \%$, and accuracy $=97.44 \%$.

Both protocols detected LM injury in 1 knee joint while arthroscopy detected it in 2 knee joints; both modalities agreed in 1 patient only and one lesion missed by MRI, reaching a sensitivity $=50 \%$, specificity $=100 \%$, positive predictive value $=100 \%$, negative predictive value $=97.37 \%$, and accuracy $=97.44 \%$. 

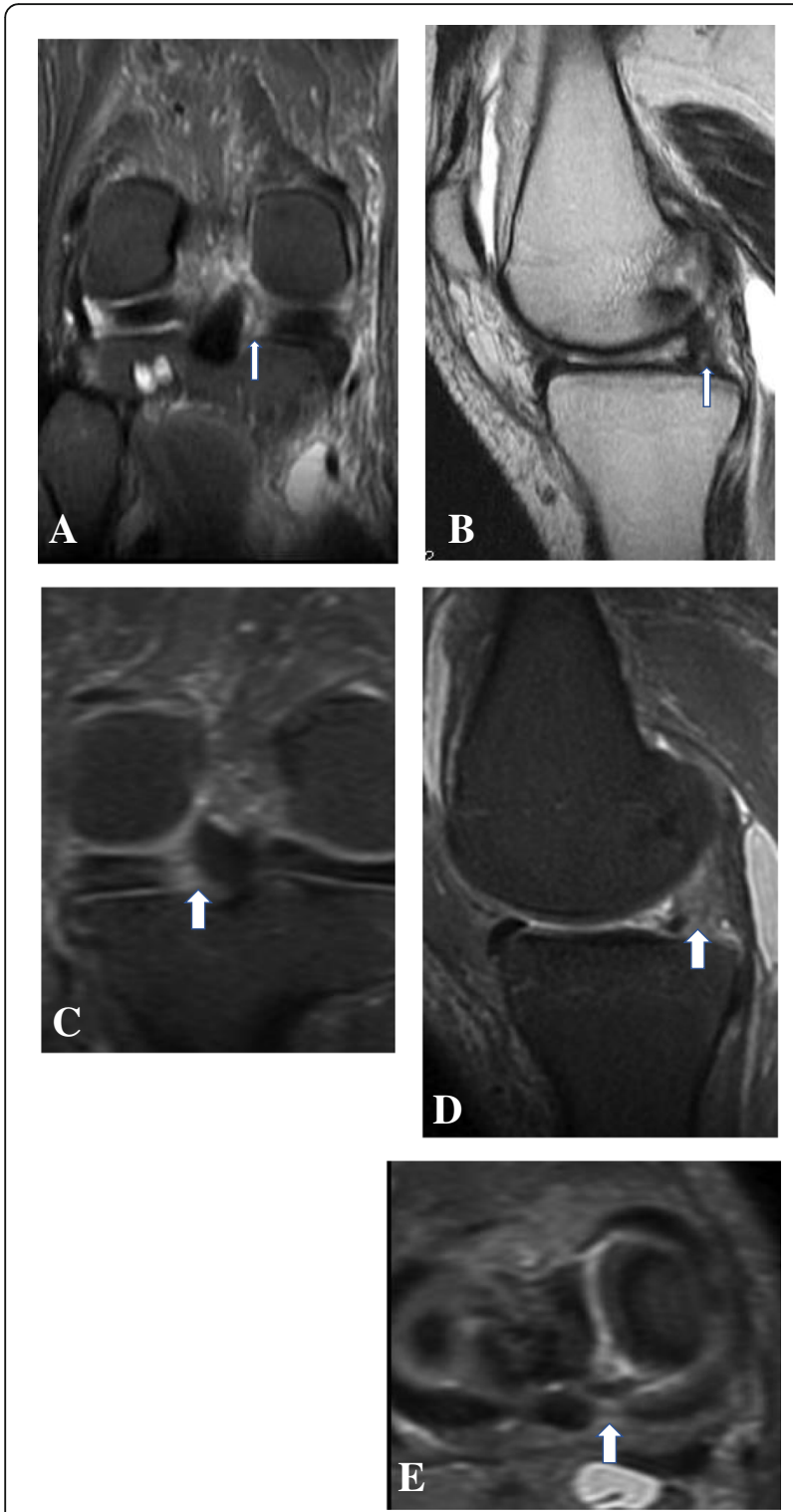

Fig. 5 A 30-year-old female patient presented by right knee pain with history of trauma. $\mathbf{a}, \mathrm{b}$ Coronal standard 2D PD WI and sagittal T2 standard 2D images, showing medial meniscus posterior root full thickness radial tear, white straight arrows. c-e 3D MPR VISTA images (coronal, sagittal, and axial), clearly outlining the posterior root full thickness radial tear, white straight arrows

Table 3 Arthroscopic findings correlated with MRI protocol A for MM lesions

\begin{tabular}{llll}
\hline & \multicolumn{2}{l}{ Arthroscopy MM } & P value \\
\cline { 2 - 3 } & Negative & Positive & \\
\hline MRI 2D MM & $N(\%)$ & $N(\%)$ & $<0.001$ \\
Negative & $26(96.29)$ & $0(0)$ & \\
Positive & $1(3.70)$ & $12(100)$ & \\
\hline
\end{tabular}

Table 4 Arthroscopic findings correlated with MRI protocol A for LM lesions

\begin{tabular}{llll}
\hline & \multicolumn{2}{l}{ Arthroscopy LM } & \\
\cline { 2 - 3 } & Negative & Positive & P value \\
\hline MRI 2D LM & $N(\%)$ & $N(\%)$ & \\
Negative & $37(100)$ & $1(50)$ & 0.051 \\
Positive & $0(0)$ & $1(50)$ & \\
\hline
\end{tabular}

\section{Cruciate lesions (Fig. 6) (Tables 7 and 8)}

Both protocols detected ACL injury in 21 knee joints which agreed with arthroscopy, having a sensitivity = $100 \%$, specificity $=100 \%$, positive predictive value $=100 \%$, negative predictive value $=100 \%$, and accuracy $=100 \%$.

There were no patients with PCL lesions either by MRI or arthroscopy.

\section{Comparative statistical analysis}

Comparing 2D MRI versus 3D MRI was done to reach accurate diagnosis by correlating the findings of each modality with the findings of arthroscopy (Figs. 7, 8 and 9).

\section{Discussion}

The main idea of Isotropic 3D MRI study is that its images can be obtained with size of $0.5 \times 0.5 \times 0.5 \mathrm{~mm}$

Table 5 Arthroscopic findings correlated with MRI protocol B for MM lesions

\begin{tabular}{llll}
\hline & \multicolumn{2}{l}{ Arthroscopy MM } & \\
\cline { 2 - 3 } & Negative & Positive & P value \\
\hline MRI 3D MM & $N(\%)$ & $N(\%)$ & \\
Negative & $26(96.29)$ & $0(0)$ & 0.333 \\
Positive & $1(3.70)$ & $12(100)$ & \\
\hline
\end{tabular}

voxels. This range of acquisition reduces the partial volume artifacts seen at conventional 2D MR images. Multi-planar reformation (MPR) may be performed in all the essential planes, including standard orthogonal planes only in a few seconds. Therefore, this technique can be helpful for the diagnosis of complex and nonorthogonally oriented internal structures of the knee [2].

Table 6 Arthroscopic findings correlated with MRI protocol B for LM lesions

\begin{tabular}{llll}
\hline & \multicolumn{2}{l}{ Arthroscopy LM } & \\
\cline { 2 - 3 } & Negative & Positive & P value \\
\hline MRI 3D LM & $N(\%)$ & $N(\%)$ & \\
Negative & $37(100)$ & $1(50)$ & 0.051 \\
Positive & $0(0)$ & $1(50)$ & \\
\hline
\end{tabular}



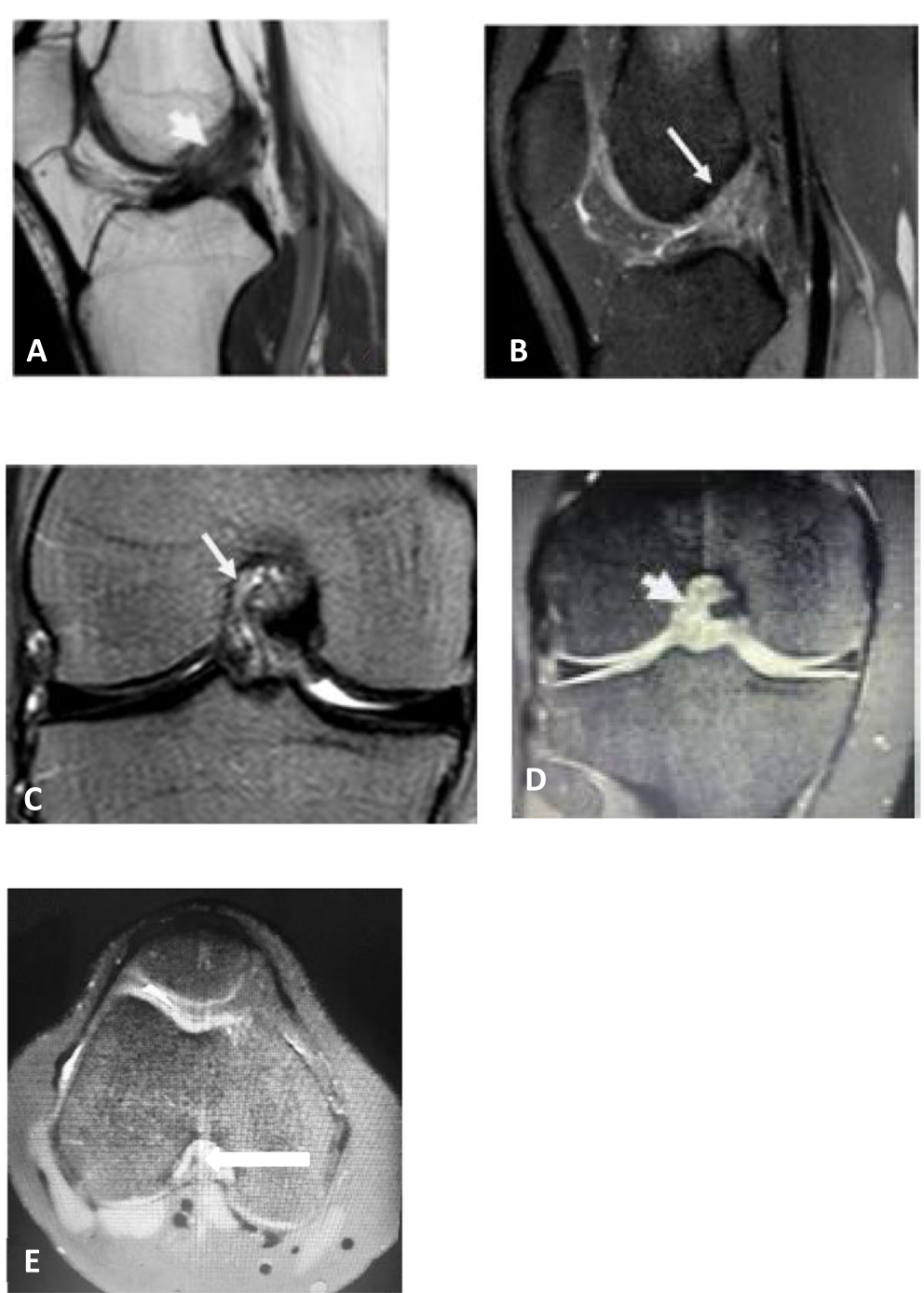

Fig. 6 A 19-year-old male patient presented by acute RT knee joint pain after trauma. a, b Sagittal Standard 2D T2WI and 3D MPR VISTA images. c, b Coronal standard 2D T2 and 3D MPR VISTA images. e Axial MPR VISTA show high-grade partial ACL tear (white arrows)

Lim et al. stated that 3D isotropic MR images accompanied by reconstructed MPR images achieve diagnostic accuracy in detection of small meniscal complex, radial, and root tears better than conventional 2D images [5]. This was likely due to small inter-slice gap as well as the capacity to reconstruct images along the important structures.

Table 7 Arthroscopic findings correlated with MRI protocol A for ACL lesions

\begin{tabular}{llll}
\hline & \multicolumn{2}{l}{ Arthroscopy ACL } & \\
\cline { 2 - 3 } & Negative & Positive & P value \\
\hline MRI 2D ACL & $N(\%)$ & $N(\%)$ & \\
Negative & $18(100)$ & $0(0)$ & $<0.001$ \\
Positive & $0(0)$ & $21(100)$ & \\
\hline
\end{tabular}

Knee MRI images have been reported as having high sensitivity and specificity for cruciate ligament lesions and meniscal tears diagnosis. Currently, most of the studies have revealed that isotropic 3D imaging technique has a diagnostic accuracy that is almost identical to the conventional 2D images $[2,5]$. The 3D VISTA sequence produces high-resolution volumetric

Table 8 Arthroscopic findings correlated with MRI protocol B for $\mathrm{ACL}$ lesions

\begin{tabular}{llll}
\hline & \multicolumn{2}{l}{ Arthroscopy ACL } & \\
\cline { 2 - 3 } & Negative & Positive & P value \\
\hline MRI 3D ACL & $N(\%)$ & $N(\%)$ & \\
Negative & $18(100)$ & $0(0)$ & $<0.001$ \\
Positive & $0(0)$ & $21(100)$ & \\
\hline
\end{tabular}

Screening test conducting using MedCalc version 18.9

Fisher exact correction done for comparison of qualitative variables 


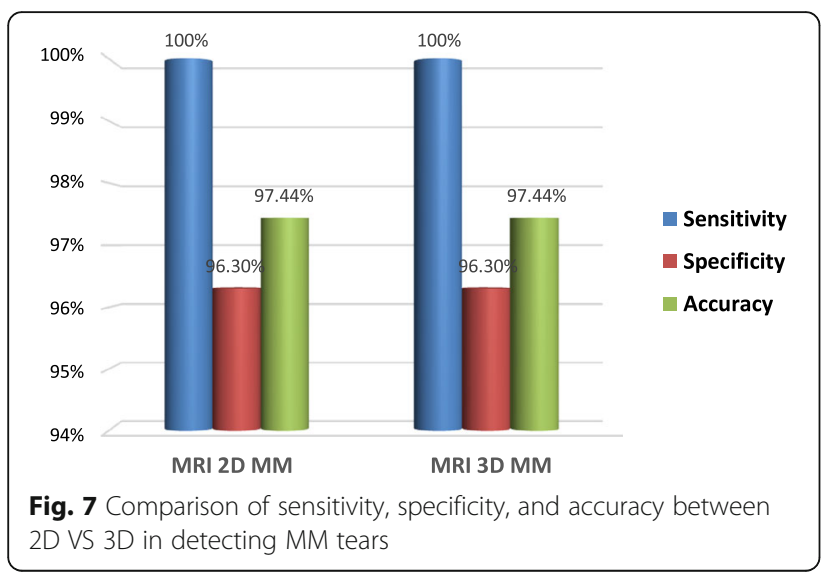

intermediate-weighted (IW) images. Inter-echo spacing and acquisition time are enhanced by utilizing reduced flip angles in combination with non-selective refocusing pulses [7].

Jung et al. compared isotropic 3D FSE and conventional 2D FSE without valid differences in diagnostic accuracy in the assessment of menisci and ligament lesions [7]. This was concordant with our results, as we also did not reach any significant difference in diagnostic accuracy for the assessment of ACL and PCL lesions and both menisci tears.

In our study, the results of both protocols, compared with arthroscopy, showed sensitivity, specificity, and accuracy of $100 \%, 100 \%$, and $100 \%$, respectively, for the diagnosis of anterior cruciate ligament tears; $100 \%, 96 \%$, and $97.44 \%$, respectively, for the detection of MM tears; and $50 \%, 100 \%, 97 \%$, respectively, for LM tears.

In a previous study, "Duc et al." agreed with us and demonstrated that their 3D sequence had similar sensitivity, specificity, and accuracy compared with conventional 2D imaging study and reported 3D fast imaging sensitivity, specificity, and accuracy of $80 \%, 95 \%$, and $90 \%$, respectively, for the assessment of anterior cruciate ligament tears; $100 \%, 82 \%$, and $90 \%$, respectively, for the assessment of medial meniscus tears; and $83 \%, 83 \%$, and $83 \%$, respectively, for lateral meniscus tears [5].

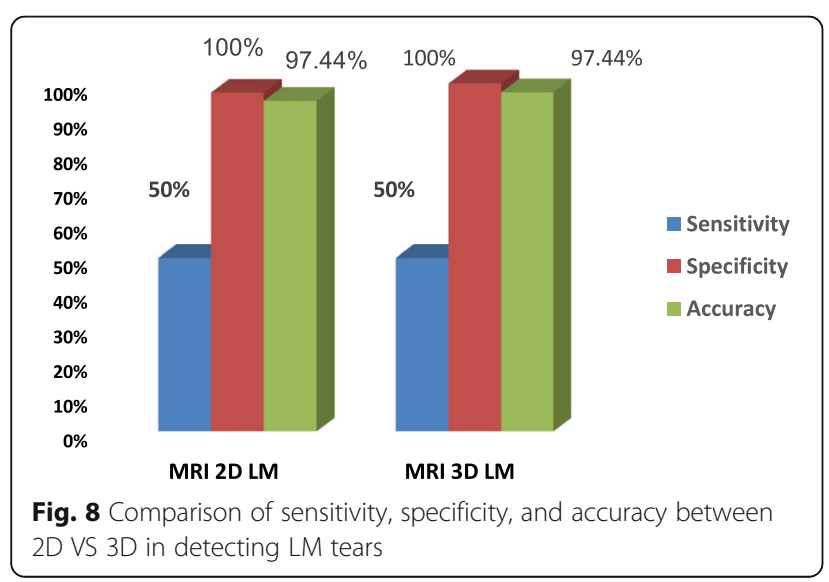

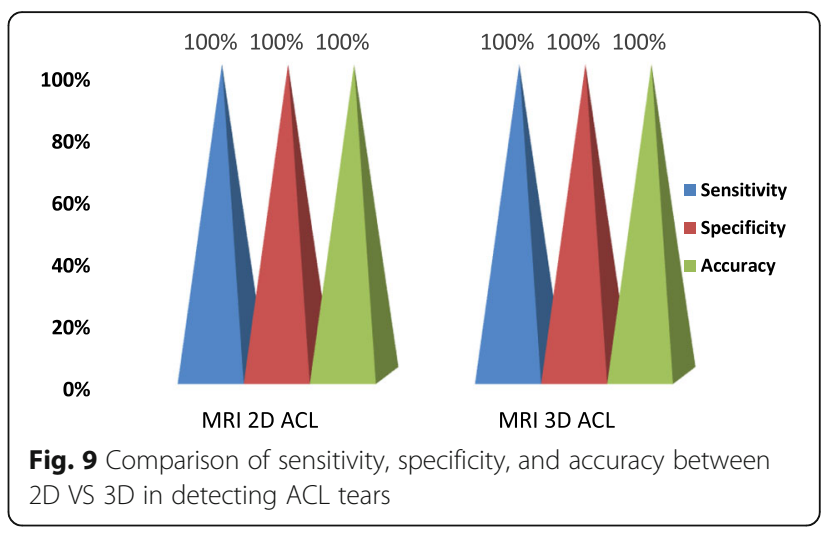

A large study by "Kijowski" was in accordance with us and stated that 3D sequence had a comparable sensitivity (90\%) and specificity (100\%) for assessment MM tears compared with standard MRI sequences [3].

In another study, "Li et al." agreed with us and demonstrated that at $1.5 \mathrm{~T}$ machine, a 3D-FSE sequence had similar diagnostic accuracy in the assessment of meniscal and cruciate ligament tears in comparison with routine 2D sequences in the orthogonal planes with accuracy $90 \%$ for MM and LM tears and $95 \%$ for ACL and PCL, but this research work was limited because the standard knee protocol was used as the reference gold standard, not arthroscopy [8]. Our study used arthroscopy as gold standard reference with net results of accuracy $97.44 \%$ for menisci and $100 \%$ for cruciate ligaments. Also, their study did not evaluate the time factor between the two protocols, and our study demonstrated that protocol A consumed $13.7 \mathrm{~min}$., while protocol B consumed $6.6 \mathrm{~min}$.

It had been documented that LM tears are more likely to be missed in conventional 2D MR images because of the LM anatomical complexity, especially if the tear affects only one third of the meniscus or found in the posterior horn [2]. Although the FS 3D VISTA sequence has isotropic voxels and smaller inter-slice gap, it did not have a better diagnostic accuracy in LM evaluation. This was in agreement with our results, as both protocols missed one case with LM tear, which was detected at arthroscopy.

The MPR images were automatically achieved in a few seconds focusing on abnormal lesions with reduced partial volume and summation artifacts and consequently increasing diagnostic accuracy. The shorter acquisition time renders the study more comfortable for the patient, which might reduce motion artifacts and improve the image quality as a concomitant effect.

\section{Limitations}

First, we had a relatively small sample (39 subjects). Second, patient choice bias may have been erroneously made because we studied only those patients who had 
arthroscopic surgery. Also, another limitation is that our patient sample did not include other ligamentous injuries, like collateral ligament injury; at the same setting, cartilage and bone marrow lesions were not investigated in the current study. Finally, based upon the results of our study, we cannot simply recommend that radiologists substitute the standard 2D FSE technique with the 3D FSE protocol for knee evaluation, because our study was confined to the menisci and cruciate ligament lesions.

\section{Conclusion}

In conclusion, our results showed that FS 3D VISTA has a diagnostic potential for the evaluation of meniscal and cruciate ligament lesions at $1.5 \mathrm{~T}$ MRI machine. The ability for time saving, with diagnostic accuracy similar to conventional 2D sequence, is important in clinical practice to achieve patient comfort.

The described 3D isotropic MR sequence produces thin-section data without inter-slice gaps and multiplanar reformatted images in orthogonal planes that are essential for the detection of complex lesions.

\section{Abbreviations}

VISTA: Volume isotropic turbo spin-echo acquisition; MRI: Magnetic resonance imaging; MM: Medial meniscus; LM: Lateral meniscus; ACL: Anterior cruciate ligament; PCL: Posterior cruciate ligament; PD: Proton density; FSE : Fast spin echo; SE: Spin echo; 2D: Two dimensional; 3D: Three dimensional; FS: Fat suppression; DICOM: Digital imaging and communication in medicine

\section{Acknowledgements}

The authors gratefully acknowledge the valuable contributions of Dr. Sherihan Salah, resident of radiology, in facilitating and performing this work. The authors are really appreciating the role of the Kasr Al-Aini Institute and Hospital for providing the study with the required type of patients and the help of reaching optimum diagnose for the proper management service.

\section{Authors' contributions}

NE conceived the study, designed it, was the primary radiologist who interpreted the images blindly from the second radiologist, and drafted the manuscript. HK participated in the design of the study and revised and adjusted the manuscript. AS was the orthopedic surgeon who made all the arthroscopies and revised the orthopedic part of the manuscript. All authors have read and approved the final version of the manuscript.

\section{Funding}

None to declare

\section{Availability of data and materials}

The datasets used and analyzed during the current study are available from the corresponding author on reasonable request.

\section{Ethics approval and consent to participate}

The study had been approved by the Research Ethics Committee of the Faculty of Medicine at Cairo University in Egypt on 201572016, in compliance with Helsinki Declaration (DoH-oct20081).

All patients included in this study gave written informed consent to participate in this research. If the patient was less than 16 years old or unconscious at the time of the study, written informed consent for their participation was given by their parent or legal guardian.

\section{Consent for publication}

All patients included in this research gave written informed consent to publish the data contained within this study. If the patient was less than 16 years old, deceased, or unconscious when consent for publication was requested, written informed consent for the publication of this data was given by their parent or legal guardian.

\section{Competing interests}

The authors declare that they have no competing interests.

\section{Author details}

${ }^{1}$ Department of Diagnostic and Interventional Radiology, Kasr Al-Aini, Faculty of Medicine, Cairo, Egypt. ${ }^{2}$ Department of Orthopedics, Kasr Al-Ainy Faculty of Medicine, Cairo, Egypt.

Received: 9 September 2020 Accepted: 15 November 2020

Published online: 14 December 2020

\section{References}

1. Kijowski R, Davis KW, Blankenbaker DG, Woods MA, Del Rio AM, De Smet AA (2012) Evaluation of the menisci of the knee joint using threedimensional isotropic resolution fast spin-echo imaging: diagnostic performance in 250 patients with surgical correlation. Skeletal Radiol 2012 41(2):169-178

2. Lim D, Lee $Y H$, Kim S, Song H-T, Suh J-S (2016) Clinical value of fatsuppressed 3D volume isotropic spin-echo (VISTA) sequence compared to 2D sequence in evaluating internal structures of the knee. Acta Radiologica 57(1):66-73

3. Kijowski R, Blankenbaker DG, Woods M, Del Rio AM, De Smet AA, Reeder SB (2011) Clinical usefulness of adding 3D cartilage imaging sequences to a routine knee MR protocol. AJR Am J Roentgenol 196(1):159-167

4. Yao L, Pitts JT, Thomasson D (2007) Isotropic 3D fast spin-echo with protondensity-like contrast: a comprehensive approach to musculoskeletal MRI Am J Roentgenol 188:W199-W201

5. Lim D, Lee YH, Kim S et al (2013) Fat-suppressed volume isotropic turbo spin echo acquisition (VISTA) MR imaging in evaluating radial and root tears of the meniscus: focusing on reader-defined axial reconstruction. Eur J Radiol 82:2296-2302

6. Lee JH, Yoon YC, Park KJ, Wang JH (2017) Diagnosis of internal derangement of the knee: volume isotropic turbo spin-echo acquisition MRI with fat suppression versus without fat suppression. Am J Roentgenol 208: 1304-1311. https://doi.org/10.2214/AJR.16.17217

7. Jung JY, Yoon YC, Kim HR et al (2013) Knee derangements: comparison of isotropic 3D fast spin-echo, isotropic 3D balanced fast field-echo, and conventional 2D fast spin-echo MR imaging. Radiology 268:802-813

8. Li CQ, Chen W, Rosenberg JK, Beatty PJ, Kijowski R, Hargreaves BA et al (2014) Optimizing isotropic three-dimensional fast spin-echo methods for imaging the knee. J Magn Reson Imaging 39(6):1417-1425

\section{Publisher's Note}

Springer Nature remains neutral with regard to jurisdictional claims in published maps and institutional affiliations.

\section{Submit your manuscript to a SpringerOpen ${ }^{\odot}$ journal and benefit from:}

- Convenient online submission

- Rigorous peer review

- Open access: articles freely available online

- High visibility within the field

- Retaining the copyright to your article

Submit your next manuscript at $>$ springeropen.com 